\title{
Virtual Product Development within a Fourth Year Option in the Mechanical Engineering Curriculum
}

\author{
B. Sanschagrin \\ C. Fortin \\ $\frac{\text { Bernard.sanschagrin }}{\underline{\text { @polymtl.ca }}} \frac{\text { Clement.fortin }}{\text { @polymtl.ca }}$ \\ $\frac{\text { Bernard.sanschagrin }}{\text { @polymtl.ca }} \frac{\text { Clement.fortin }}{\text { apolymtl.ca }}$ \\ A. Vadéan \\ A. Lakis \\ Aurelian.vadean \\ Aouni.lakis \\ École Polytechnique de Montréal, Department of Mechanical Engineering
}

\begin{abstract}
The Mechanical Engineering Department of École Polytechnique after experimenting for a number of years with a virtual environment option in the Aerospace Master Program, created a new design option in the fourth year of the Mechanical Engineering program. This option is built around a project carried out by a large team working over two semesters where a digital product definition including Product Data Management and Manufacturing Process Management technologies are currently used to foster a concurrent product development process.
\end{abstract}

This orientation contains three courses and the project. A series of laboratory sessions have been developed in each course in order to familiarise the students with the virtual environment and also the various types of analysis that they will have to perform on the product being developed. Professors teaching the three courses participate in the project definition and the project assessment. This approach reinforces the various subjects' knowledge and integrates it into a practical realm close to an industrial environment.

The project assessment includes four reviews. A requirements review and a conceptual review are set in the first semester and a preliminary design review and a critical or detail design review in the second semester. At each review, the students prepare a technical report followed by an oral presentation to a jury composed of four professors of the option, engineers from industry and the professor coordinating the SAE Student Formula. At each review, the students have to evaluate the work completed by each participant to the project. This evaluation impacts on the individual assessment of the project.

The vision for this project is to integrate practical building and test experiences by coupling the option courses with an already existing lab course in the last semester. A major part of this lab course is oriented for practical team training (3 - 4 students) on a given number of laboratory experiments. It is planned that some of these labs will be focused on the analysis and testing of subassemblies already designed and built during the project of the previous or the current year. This goal is in line with our CDIO (Conceive, Design, Implement, Operate) initiative which aims to include in the engineering curriculum a number of design, build and test experiences.

\section{Introduction}

The implementation of a virtual product development environment supports the modification of an existing design and analysis option. This modification aims at developing, during the last year of the Mechanical Engineering curriculum, a more realistic environment representing the future engineer's work. The starting point of this modification comes from the expertise developed in a Master Degree in Aerospace option where such a virtual environment was introduced 7 years ago. This aerospace option, which focuses on the product development process and soft skills, was developed in collaboration with the aerospace industry, namely Bombardier Aerospace, Pratt\&Whitney Canada and Bell Helicopter Textron and contains a project associated with two courses where academics and engineers from industry are collaborating. This option has allowed us to build a virtual environment laboratory simulating the engineer's workspace in the aerospace industry. IBM Canada provided strong support initially for the product definition system and in the last two years, Polyplan Technologies provided support for the Manufacturing Process Management (MPM) system. This experience has run since 1998 with great success based on comments from the students and also the employers. The initiative won the Conference of Canada Prize for Industry and University Collaboration in 2001. 
From this experiment, we decided to transpose this concept at the Bachelor level of the Mechanical Engineering program. To do so, we modified an existing option of 12 credits in the last year of the curriculum. This option called an "orientation" contains three courses associated to a project.

The structure of the design and analysis option is presented in figure 1. The first course MEC6508 familiarises the students with the virtual environment while the second course MEC4410 insists on the design of 3 and 4 bars mechanisms and the dynamic balancing of rotating systems. The first part of the project deals with the problem definition and the elaboration of various concepts to solve the problem. In the following semester, students take the third course MEC4320 on advanced design of mechanical components where they learn to optimize a given design using tools such as Ansys and Ansys workbench. At the same time, the project deals with the preliminary design and detailed design phases of the design methodology.

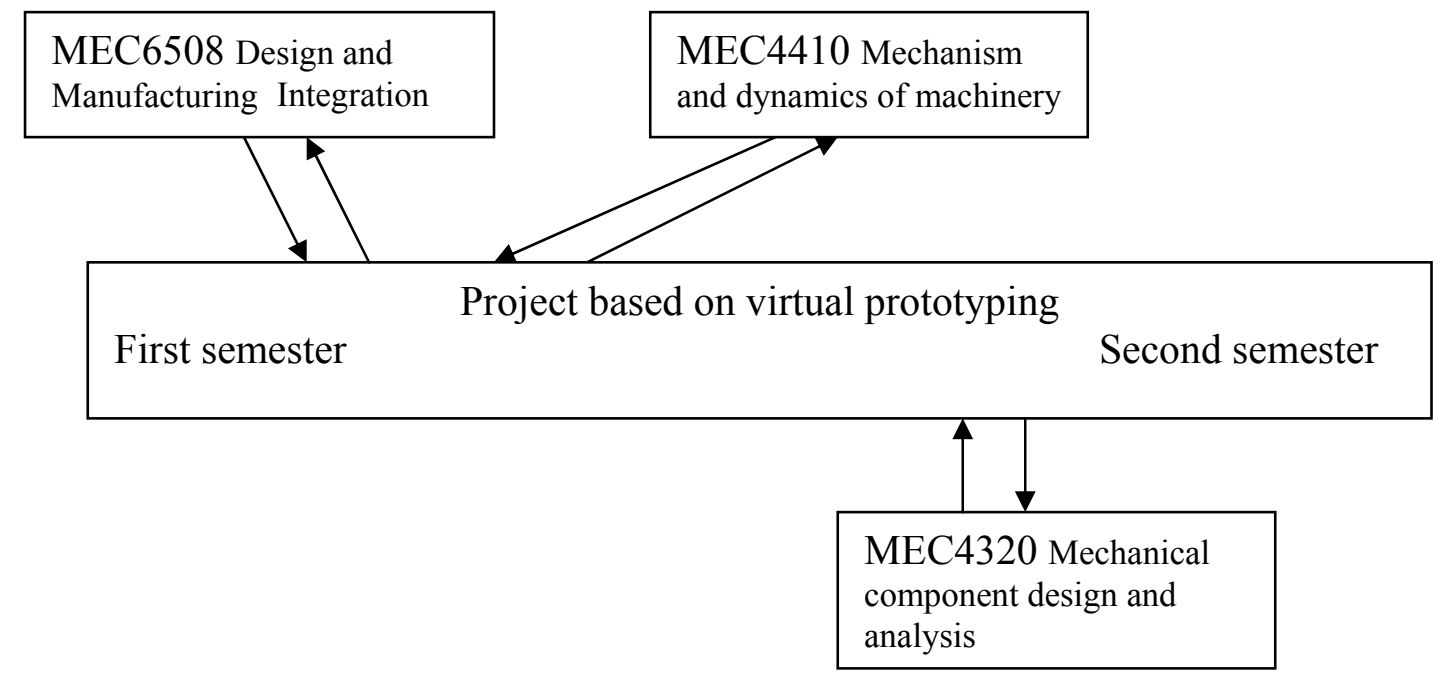

Figure 1. Structure of the design option

\section{Option Courses}

The three courses have been tailored to bring the fundamental notions in a just in time approach with the project.

\section{Integration of Design and Manufacturing}

In the course MEC6508, the students learn how to use the virtual environment to support the complete product development process. They learn how to build and manage a virtual product using specific tools such as CATIA V5 supported by a Product Data Management system (PDM), Enovia VPM in this case. This system supports the various steps a complete product passes through during the design phases with the various approvals until the release of each part and sub-system. This system contains the various roles and responsibilities related with the engineering functions such as designers, supervisors, configuration manager and project manager. A lot of emphasis is placed on the development of technical and business process methodologies to support the development of a complex product with a large team of engineers. The change process and configuration management issues are key elements of the subject.

In addition to these tools, the environment laboratory contains also an MPM system, a tool developed from our own internal research and now in full industrial production on very complex products, to define and manage the product manufacturing. This system supports the manufacturing process definition of a complete product including the various approvals until the release of the product. The system is integrated with the CAD/PDM system and supports for a large development team the concurrent product and manufacturing process development. It supports the definition of all types of process plans for very 
complex products such as airplanes, trains and cars. Here again many roles and responsibilities are distributed in the manufacturing team responsible for the fabrication of the product.

In this course, the students learn the notion of product and manufacturing systems, complementary to each other, and containing all the information related to a specific product. They also learn how to organise this information, how to verify it and concurrently work on it. In practice, they learn how to build an engineering bill of material (EBOM), which contains all the engineering information on all parts of a product. This information covers all kinds of specific information such as the $3 \mathrm{D}$ representation of a part and assemblies, 2D technical drawings, various types of analyses such as structural, thermal or heat treatment, etc. Other documents associated to a part, such as engineering changes and so on, can also be managed.

Students also learn how to build a manufacturing bill of material (MBOM), which also contains all the manufacturing information on all parts of a product. The MBOM is build from the EBOM but is not structured in the same fashion. In a concurrent scheme, the manufacturing engineers start to work on the fabrication of the parts before the engineering product is completely released, but this needs a high communication level between the two groups of engineers.

In the course MEC6508, students take several laboratory sessions where they learn how to use the virtual environment, including roles and responsibilities with very simple products such as the one presented in figure 2 . Then, they have to apply these principles and systems on a more complex product in the project. The role of the virtual environment is essentially to create a product development environment of industrial complexity within an academic setting.

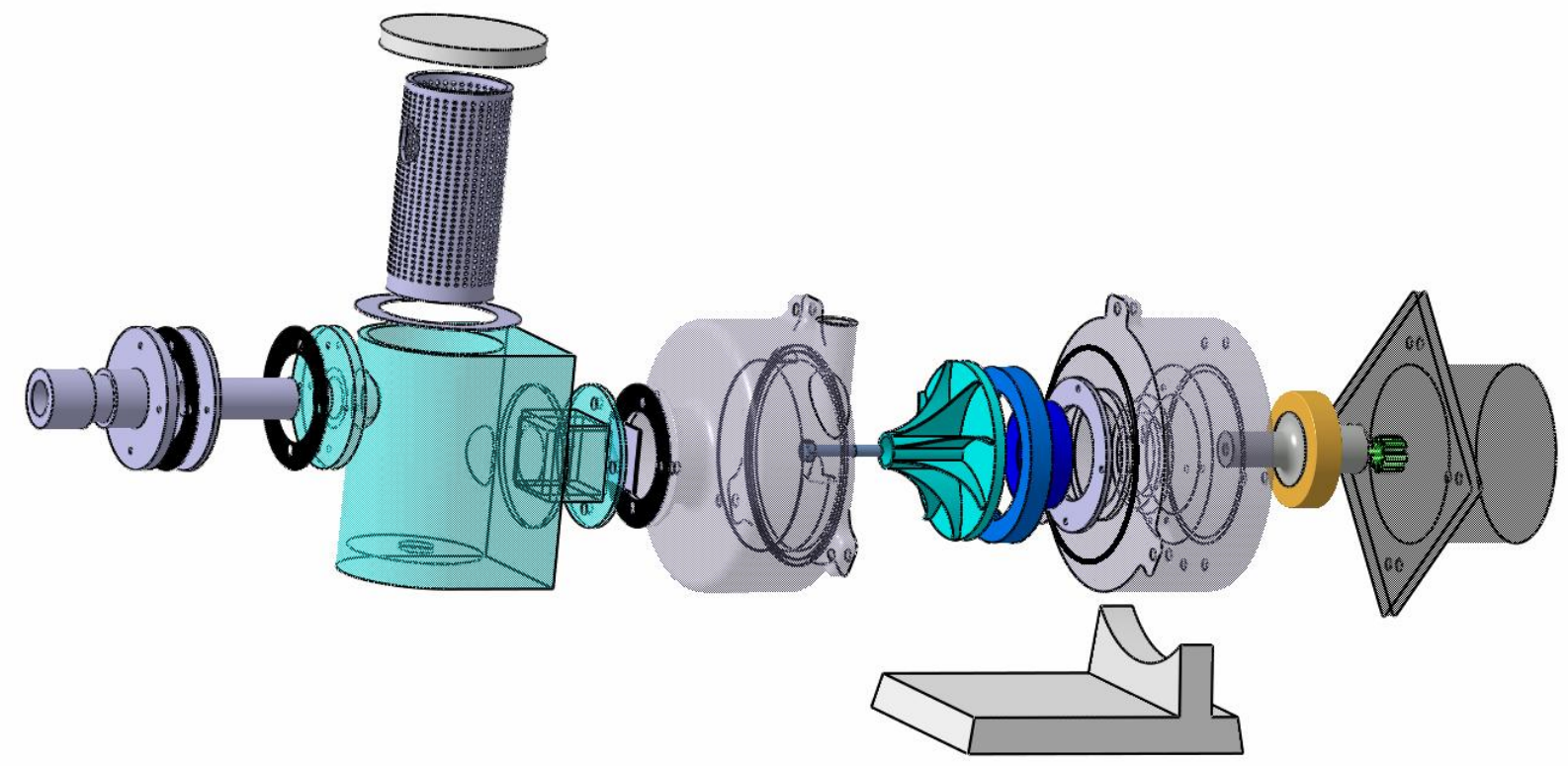

Figure 2. Exploded view of a pump

\section{Mechanism and Dynamics of Machinery}

The course MEC4410 (Mechanism and Dynamics of Machinery) has been transformed in order to adapt it to the new design option. This course consists of 3 hours a week lecture for 13 weeks and 3 hours of project a week during 7 weeks.
During the summer of 2004, we reorganised and adapted the course project in order to be in tandem with the capstone project of "Virtual prototyping".

While this course attempts to be thorough and complete on the topics of analysis, it also emphasizes the synthesis and design aspects of the subject to a 
greater degree than most textbooks. It also emphasizes the use of computer aided engineering as an approach to the design and analysis of this class of problems by developing and providing software that can be used to design vehicle components and to balance rotating machinery.

Unstructured, realistic design problems which have many valid solutions are assigned. Our intention is that some synthesis topics be introduced first in order to allow the student to work on some simple design tasks early in the term while still mastering the analysis topics.

Though, this is not the "traditional" approach to the teaching of this material, we believe that it is a superior method compared to focusing on the detailed analysis of mechanisms for which the students have no concept of the origin or the purpose.

We found out, after the first year, that the assignment and execution of unstructured small projects can greatly enhance the student's understanding of the concepts.

\section{Mechanical Components Design and Analysis}

The third course of the Design option, MEC4320 Mechanical Components Design and Analysis is offered in the winter term which corresponds to the second part of the virtual prototyping project.

The main objective of this course is to complete the background on the design principles presented within a previous course on machine elements called Transmission of Power.

Moreover, the presentation of new concepts and the use of 3D finite elements analysis were introduced in order to perform in-depth analyses, functional and geometrical optimizations. Exercises in communication skills and team organization for results driven projects are also particular goals of this course.

To achieve this objective, a series of practical exercises are proposed to train the students on the analysis and the optimization environment which is ANSYS Workbench. The functionalities of various modules of the software (Design Modeller parametric modeling, Simulation - 3D Finite Element analysis and Design Explorer - optimization) are presented through relatively simple examples: analysis and optimization of a drive shaft, dynamic analysis of a rotor of an electrical generator, fatigue analysis and verification of a bolted joint for a cable cabin support. For all these studies, comparisons are carried out between the results of the analytical calculations and those of the numerical results (FEA).

Figure 3 presents the case study of the driveshaft. Calculations are initially performed by using the traditional method of strength of materials. The critical section of the shaft is highlighted by the classical computations and the safety factors are calculated according to the appropriate failure theories.

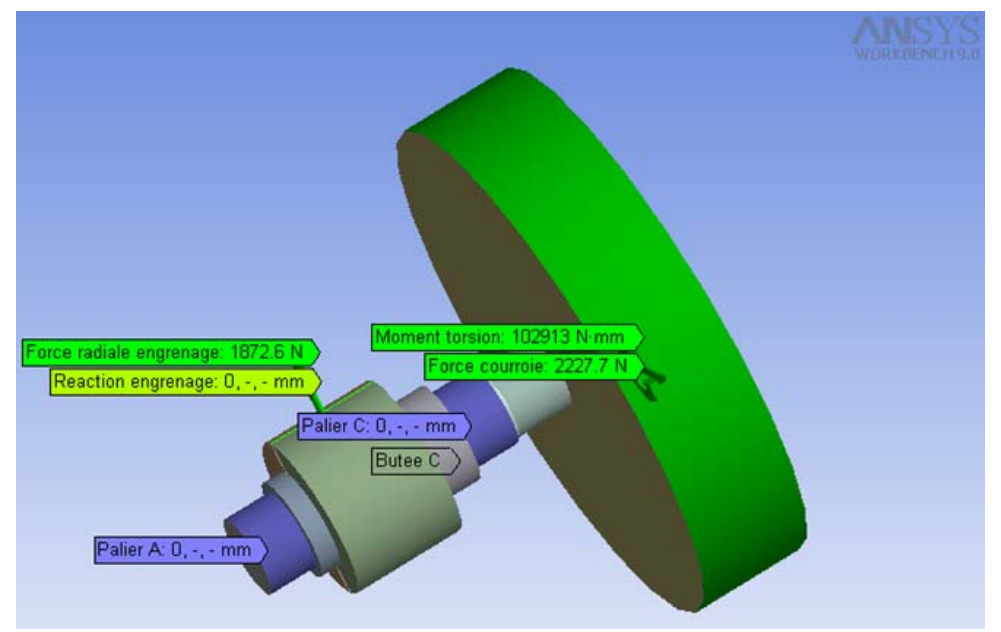

Figure 3. Shaft assembly modelling: gear and pulley, bearing supports, forces and torques 
Compared to the analytical pre-design, the 3D FEA analysis can take into account the contributions to the global stiffness of different components assembled on the shaft (gears, pulleys) as well as the specific bearing supports. A methodology based on parametric modeling and specific fatigue analyses according to the stress type (steady torque and fully reversed bending) was established.

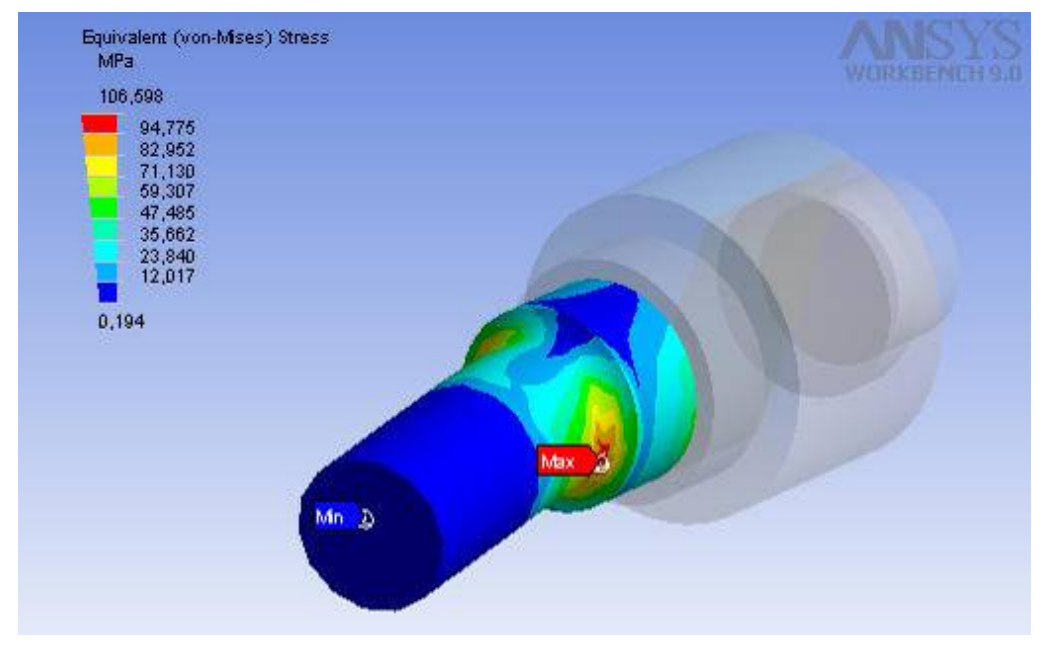

Figure 4. Maximum stress after optimizing shaft diameter and fillet radius

The Design Explorer module of ANSYS Workbench and its Design Of Experiments (DOE) module are used to determine the allowable design space according to the variations of the input parameters (i.e. shaft diameter and fillet radius on the critical zones), the output parameters (i.e. different types of stress) and the derived parameters (i.e. safety factors). An example of this type of analysis is shown in Figure 5.

\section{Fatigue Safety Factor}

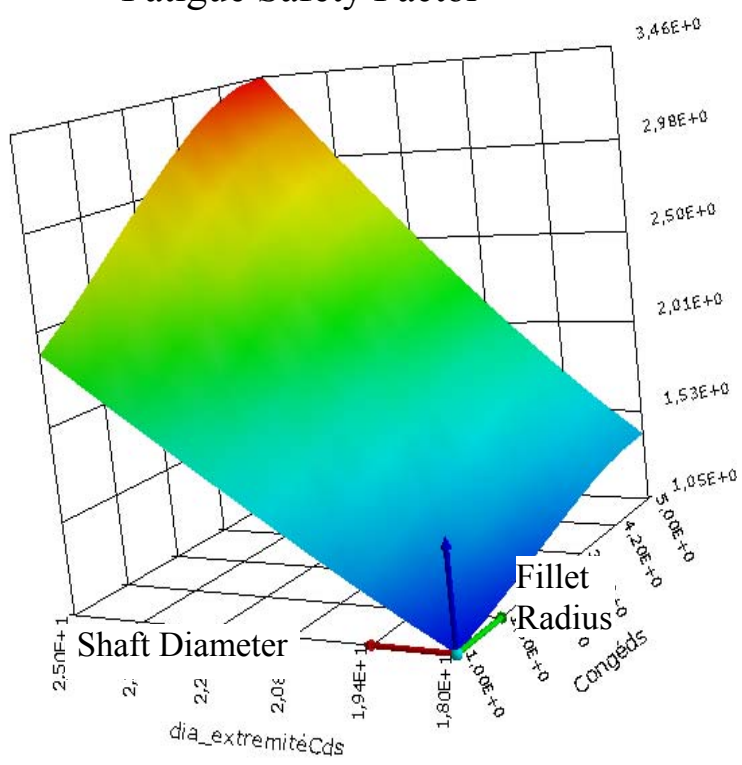

Figure 5. Design space determined by DOE 
Finally, a Goal Driven Optimization (GDO) is applied to choose the best candidate with an automatic regeneration of the parametric model.

All the laboratories are performed by groups of two students. The handling of analytical and numerical techniques and, of DOE is essential for the second part of this course where an in-course project is carried out by a team of 4 to 5 students.

A great majority, in the order of $80 \%$, of the themes of the in-course project are directly related to the capstone project of the option, the Formula SAE. Here are some examples of the chosen themes: analysis of the torsional rigidity and of the side impact behaviour of the car frame, analysis of the rear suspension (up-right member and axle), dynamic analysis and damping of the engine mounts, design optimization of the wheel drive shaft and the study of the front and rear suspensions and their component rods. The constitution of the teams, the organization of each one, the task distribution and the establishment of a working plan are the total responsibility of the students.

It is important to emphasize that, on the one hand, the theoretical and technical knowledge of the present course is directly used to address the needs of the capstone project; on the other hand, the personal and organisational skills, as well as all the concepts of team work, learned and developed within the courseproject, are used in this particular course to successfully carry out the in-course project. Consequently, this gives us the opportunity to realise a complete exchange of knowledge and personal skills between the two courses.
The assessment of the in-course project was carried out at various stages and with various weights: $40 \%$ by peer assessment at half term, followed by a $20 \%$ weight for the final presentation and the last $40 \%$ comes from the report assessment by the professor. The half term peer assessment was extremely useful, since it generated discussions on the project and also provided written recommendations of the judges to each team. Furthermore, it was also an excellent opportunity to be acquainted with each other's work, their difficulties and successes. Multiple software tools are used to address the different design and analysis needs: CATIA V5, ANSYS Workbench, ANSYS classic, Simulink, MSC ADAMS, MATLAB, AutoCAD and Excel spreadsheets.

As difficulties were encountered, it was noticed that the goals were too ambitious for the resources and knowledge involved. Moreover, the relatively broad sweeping themes called for a close supervision from the professors, various specialists in industry and hotlines for software support. Adjustments must be made to the course content to alleviate these difficulties.

As an example, one of the in-course projects was the optimization of the car frame. Several design steps were performed in order to reach the maximum torsion stiffness and to provide maximum protection for lateral impact. The main goal of this study was to attain the maximum rigidity as accurately as possible. The importance of this work is obvious for characterizing the general track behaviour of the car and also to determine the load transfer in order to design and tune the suspension. Figure 6 presents the final optimized version of the car frame where steel and composite side panels were adopted. 


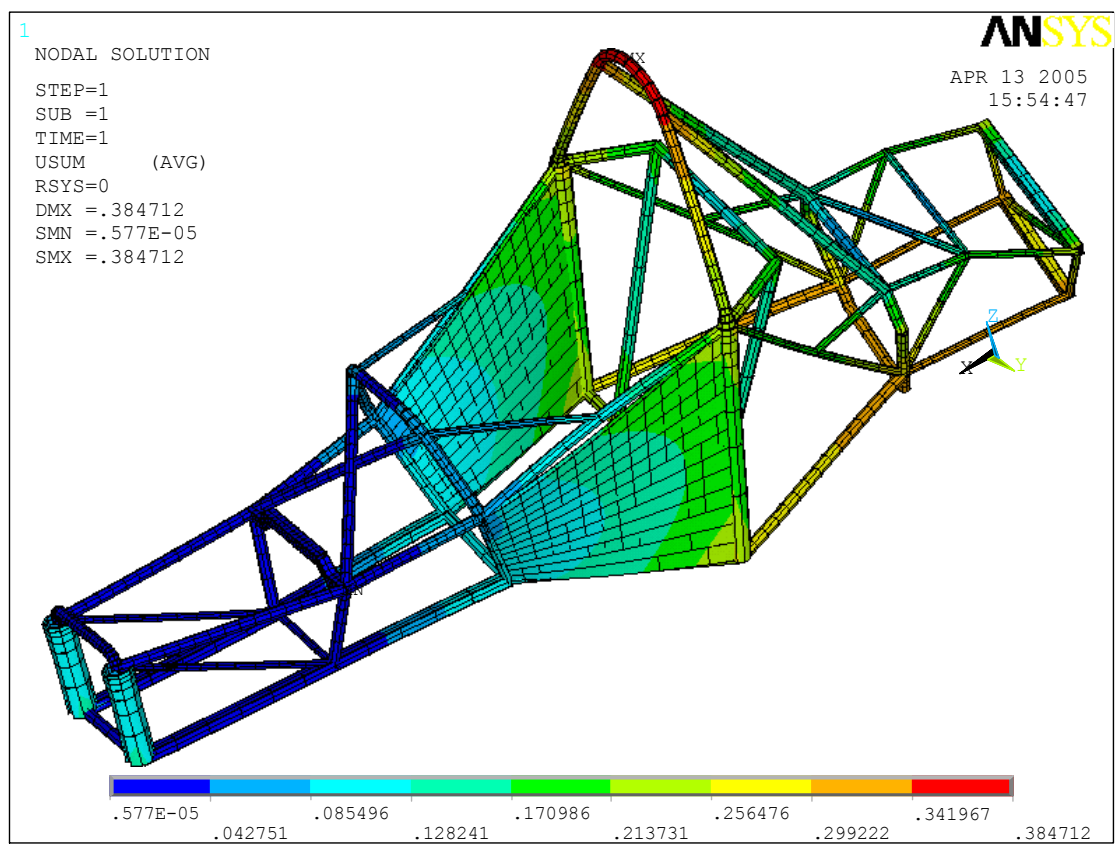

Figure 6. Optimized tubular frame with composite side panels

\section{Capstone Project}

The project MEC4340 is a capstone project realised in the last year and split on 2 semesters. In 20042005 , this project, which is based on our previous experience in the Aerospace Master program, was offered for the first year. There were 19 students which were all involved in the same project. At the beginning of the first semester, the supervisor of the project with each of the professors involved in the three courses of the option presented the project to the students. Two main documents are given:

$>$ the Formula SAE rules for the 2005 competition (new car) and the Formula student 2005 (modification of the 2004 prototype) which are similar to a technical requirement document which define the context where the product will be used, the time frame to design and build a prototype and all the security aspects that have to be met;

$>$ the statement of work defining that, during this project, there will be four design reviews where students will have to prepare a report and make oral presentations to the professors involved in the design option.
At this first meeting, the students are given the MBTI assessment; these Myers-Briggs results help them for the team set-up. During the next three weeks, the students have to prepare the requirements review where they have to read the documentation, look at the results obtained by the various teams on the preceding years of competition, decide how they will organize themselves to conceive, design and build a new virtual prototype.

In the requirements review, the students have to identify the conflicting or missing information and to present the characteristics of the existing systems or prototypes. Then, they will present an overall schedule for the project and a detailed schedule for the next phase until the conceptual review. They must describe the complete organisation of all the students in order to complete the statement of work, including the project manager, how many teams they will form and the major roles and responsibilities for the project execution. For example, this year the 19 students decided to divide into three teams, one who is responsible for the modification of the Formula student 2004 and two other teams for the Formula SAE 2005, one for the suspension, steering and chassis and the other for the engine, power transmission and accessories such as electrical and electronics, fuel system and other components. 
In each team, there is a team leader and each member of the team assumes a responsibility for the duration of the project and becomes a specialist such as: mock-up definition, dynamic analysis (ADAMS), structural analysis (ANSYS), external information (standard parts, standards, and general documentation), fabrication, suspension design, chassis design, power transmission, reliability, risk assessment, configuration management and other tasks.

A budget is put in place where they will take into account the time consumed on the project with all the expenses for consulting, part and subsystems costs, manufacturing cost, etc.

The second review is the conceptual review where the students have to present the follow-up of the requirements review; it includes how they take into account the questions or comments that have been made at the last review from the professors, adjustments on the project schedule and budget. They present the various concepts explored with their advantages and disadvantages and the criteria used for the selection of the most interesting concepts. They also present a risk assessment with identification of the major risks and their mitigation.

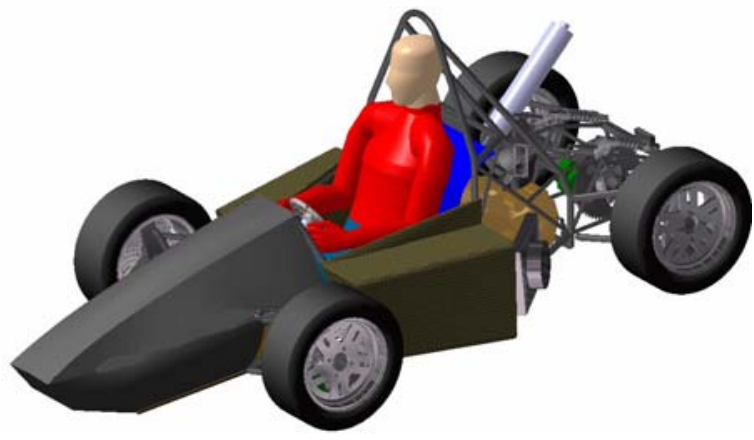

In the second semester, they work on the preliminary design review where they have to present the followup of the conceptual review. A complete solution of the problem is presented with all the justifications for the decisions made. A configuration methodology is presented and discussed. A first estimation of the product cost and weight is made and analyses of critical parts are presented.

At the end of the second semester, a detailed design review with the final report is presented to the professors. This report contains a follow-up of the preliminary design review, the Catia mock-up of the complete formula with the EBOM and MBOM, the complete analysis of the main parts of the product such as the suspension, the chassis, the engine and transmission, etc.

A complete production proposition for the whole prototype and production vehicle with identification of the critical factors and finally the final budget of the project are presented. For example in the following figures, some typical results obtained by the students are presented.

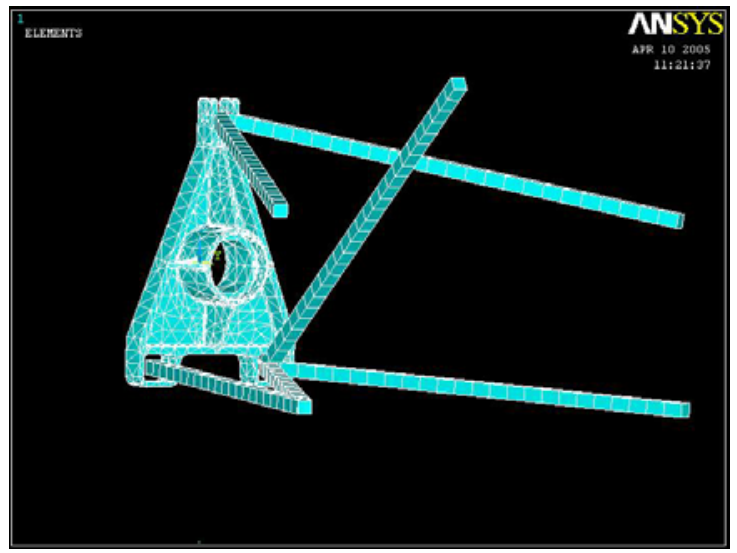

Figure 7. The mock-up of the Formula and an analysis of the up-right 


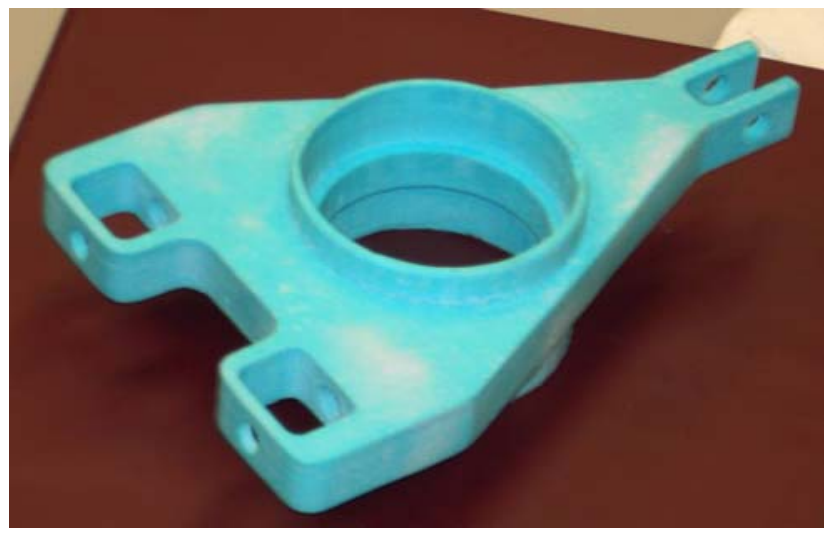

Figure 8. Rapid prototyping of the up-right

\section{Evaluations of project}

As the project is a team effort, all the evaluations made by the professors are group evaluations and they are divided in two categories:

- for the four oral evaluations, one at each review, each student participating to the presentation is individually evaluated by each professor and he receive a synthesis of the evaluations with the various comments made by the professors; but all students of the project received the same result for the presentation which is the mean of the ratings distributed by the professors. Then the students who are not presenting, participates to the preparation of the presentation by criticizing their team member during the dry run of the presentation and the final result is also a consequence of their contribution. During the project, all the students must present at least once and the students are encouraged to use their own strategy to obtain the best results.

- For the four reports, given to the professors at least 4 days before the presentation, each professor fills an evaluation sheet. The students received a synthesis of the evaluations with all the comments made by the professors. The final mark for the report corresponds to the mean evaluation. In addition to that, each student evaluates all the other students participating to the project. The students receive a synthesis of these evaluations. The weight of the rating given by a student, as evaluator, is related to the role he plays within the team. The relation the evaluator also has with the student who is evaluated plays a role. To illustrate this, three examples are presented. The organisation has been divided into three teams but all the students are working on the same project which is a Formula SAE design. For a team member evaluating a student of another team, the weight of his evaluation is one. For a team member evaluating a student member of his team, the weight of his evaluation is two. For a team leader evaluating a student member of his team or the project leader evaluating any student of the project, the weight of his evaluation is three.

Then for each evaluator, all his weighted evaluations are compiled and the mean of these evaluations is calculated. Then each evaluation given can considered as a plus if the evaluation is higher then the mean and a minus if the evaluation is lower then the mean. Now for each student, the pluses and minuses are compiled and give a representation of the individual effort of each student to the collective work or report. For example, if the compilation gives a +1 , that means the peers consider that this student contribution to the collective work is higher then the mean contribution and the final mark for this student for the given report will be the mean result given by the professors plus one. If the compilation gives zero, this means that this student contribution corresponds to the result given by the professors. If the compilation is -1 , means that the peers consider this student 
contribution to the collective work is lower then the mean contribution and the final mark for this student will be the mean mark given by the professor minus one.

Normally, after a review, there is a meeting where the students discuss what has been well done and what has to be improved in the next phase of the project. These points cover the technical project as well as the organisation, the team work, the communication, the peer evaluation and everything related to the advancement of the project.

We noticed that this approach increases the autonomy and responsibility of the students within the project but there is also a drawback. Some students don't like to be evaluated by their peers, they always have been evaluated by the professors and they don't believe that other students have the competency to evaluate their work. Very often, the students in a classroom are in a competition mode rather then a collaboration mode for the best marks. However, this is the reality of the professional life.

\section{Conclusion}

After the last presentation, the students comment on the project, realise all the work they have done on such a complex project, how this project urged them to return to some of the concepts and theories they have learn in various courses during the curriculum and how they are able to deal with that material to take decisions and reach the technical goal. They appreciate the fact, that generally they take their responsibility when they are put them in a situation where this is a clear objective to reach. At the end of the second semester, the 19 students have been able to present a collective synthesis document of 100 pages that describe their solution, their analysis and also their construction where every member of the team participates in the realisation of one part of this team project. This was the objective of the original virtual development project which was not so virtual after all.

\section{Acknowledgments}

This initiative has become feasible because of the strong financial support from the Centre d'adaptation de la main-d'oeuvre en aérospatiale du Québec (CAMAQ) which funded the virtual environment laboratory. The internal support of the Fonds de renouvellement des approches pédagogiques dans les programmes (FRAPP) is also acknowledged. This program provided major financial support to develop and adapt the various laboratory sessions in each of the three courses. It also contributed to improve the collaboration between the professors involved and to refine the evaluation process of the project. 